\title{
Kerr frequency combs and the triangular spectra
}

\author{
Zheng Liu ${ }^{1}$, Saliya Coulibaly ${ }^{1}$, Majid Taki ${ }^{1, *}$, and Nail Akhmediev ${ }^{2}$ \\ ${ }^{1}$ Université de Lille, CNRS, UMR 8523 - PhLAM - Physique des Lasers Atomes et Molécules, F-59000 Lille, France. \\ ${ }^{2}$ Optical Sciences Group, Research School of Physics and Engineering, Institute of Advanced Studies, Australian National University, Canberra ACT 0200, \\ Australia \\ *Corresponding author: abdelmajid.taki@univ-lille1.fr
}

Compiled August 2, 2021

\begin{abstract}
Nonlinear externally driven optical cavities are known to generate periodic patterns. They grow from the linearly unstable background states due to modulation instability. These periodic solutions are also known as the Kerr frequency combs which have variety of applications in metrology. Stationary state of periodic wave trains can be explained theoretically only in weakly nonlinear regimes near the onset of the instability using the order parameter description. However, in both weakly and strongly nonlinear dissipative regimes, only numerical solutions can be found. No analytic solutions are known so far except for the homogeneous continuous wave solution. Here, we derive an analytical expression for the intra-cavity fully nonlinear dissipative periodic wave train profiles that provides good agreement with the results of numerical simulations. Our approach is based on empirical knowledge of the triangular shape of the frequency comb spectrum. ( 2021 Optical Society of America
\end{abstract}

OCIS codes: (070.5753) Resonators; (190.4370) Nonlinear optics, fibers; (190.3100) Instabilities and chaos; (190.5530) Pulse propagation and temporal solitons.

http://dx.doi.org/10.1364/ao.XX.XXXXXX

Frequency comb generators have emerged as powerful tools for high-resolution metrology [? ]. Frequency standards, synchronization of atomic clocks, measurements of fundamental constants, modern astronomy and digital telecommunications rely on these devices [? ]. Initially, ultrafast mode-locked lasers were the main sources of frequency combs [? ? ]. Nowadays, a large variety of optical devices are developed to produce them [? ? ? ? ]. The most popular ones are based on continuously pumped optical cavity containing a Kerr medium [? ? ? ? ]. Known as "Kerr frequency combs" [? ], they are essentially periodic trains of optical dissipative solitons [? ? ]. Generally speaking, dissipative systems exhibit complex dynamics revealing variety of structures: fronts [? ? ], solitons [? ? ] and periodic formations or rolls [? ? ]. Here, we consider only dissipative periodic solutions. The initial growth of periodic wave trains is usually well described in terms of a linear stability analysis of stationary continuous wave solution of the system. However, this technique is absolutely insufficient for describing further stages of pulse train formation. Therefore, multi-scale analysis can be used to derive the order parameter equations describing the nonlinear dynamics of periodic structures [? ]. Recently, it has been shown that the order parameter description can be extended to the second and third harmonics of most of the amplified modes [? ]. Moreover, both theoretical and experimental spectra reported in [? ] clearly show that the output is composed of a relatively large number of harmonics. The most noticeable feature of the spectra is their triangular shape in semi-logarithmic scale for a wide range of the system parameters. This triangular shape of the spectra is tightly related to modulation instability and periodic solutions of the nonlinear Schrödinger equation [? ? ? ]. It is hardly surprising because most of the micro- or fibre cavities consist of nonlinear conservative medium with the pump provided externally. Therefore, finding connections between the known analytical results for integrable systems and periodic solutions for pumped cavities would be highly useful. Speaking generally, in order to construct fully nonlinear solutions of these devices far from the threshold, the inclusion of all spectral harmonics in the analysis is necessary.

In this letter, we propose to take advantage of the triangular shape of the spectrum to construct an analytical description of the fully nonlinear stationary periodic states of an all-fiber optical ring cavity. For this purpose, we have computed the amplitudes of all successive harmonics around the central, most amplified, frequency. The generic triangular shape of the spectrum enabled us to construct the analytic expression which accurately describes the fully nonlinear periodic solution.

The system under investigation is a passive cavity that consists of a Kerr medium externally driven by an optical light source as depicted in Fig. ??. This Kerr cavity system can be modelled by the extended nonlinear Schrödinger equation that describes the propagation along the medium supplemented with boundary conditions [? ? ]:

$$
\begin{aligned}
\partial_{z} A(z, T)= & -\alpha_{f} A(z, T)+i \sum_{n \geqslant 2} \frac{i^{n} \beta_{n}}{n !} \partial_{T}^{n} A(z, T) \\
& +i \gamma A(z, T) \int_{0}^{\infty} R\left(T^{\prime}\right)\left|A\left(z, T-T^{\prime}\right)\right|^{2} d T^{\prime} \\
A\left(0, T+T_{R}\right)= & \sqrt{\theta} E_{i}(T)+\sqrt{\rho} A(L, T) e^{-i \Phi_{0}} .
\end{aligned}
$$

Here $T_{R}$ stands for the round-trip time which is the time taken by the pulse to propagate along the cavity with the group velocity, $\Phi_{0}$ is the linear phase shift, $\theta(\rho)$ is the mirror transmission (reflection) coefficient, and $L$ is the cavity length. The electric field amplitude inside the cavity is $A$. Each of the coefficients $\beta_{n}$ is responsible for the $n$-th order dispersion, $\gamma$ is the nonlinear coefficient, and $\alpha_{f}$ is the attenuation along the fiber. Independent variable $z$ is the longitudinal coordinate while $T$ is the time in a reference frame moving with the group ve- 
locity of the light, and $R(T)$ is the nonlinear response including both instantaneous (Kerr effect) and delayed contributions (Raman effect).

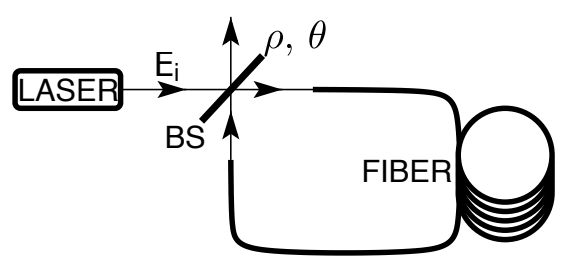

Fig. 1. Scheme of the fiber ring cavity with external pump. Here, BS is the beam splitter, $\rho$ and $\theta$ are the reflection and transmission coefficients of BS with $\rho^{2}+\theta^{2}=1$.

For the sake of simplicity and straightforward mathematics, in what follows, we neglect all the dispersion effects except for the second order one, and we also assume that the intra-cavity field is slightly depleted over one round trip (mean field approximation). We also restrict the nonlinear response to its instantaneous part. Under these assumptions, the evolution of the electric field inside the cavity is well described by the Lugiato-Lefever equation (LL model) [? ? ]:

$$
\frac{\partial \psi}{\partial t}=S-(1+i \Delta) \psi-i \eta \frac{\partial^{2} \psi}{\partial \tau^{2}}+i|\psi|^{2} \psi
$$

where we have set $S=E_{i} \sqrt{\gamma \theta L / \alpha}, \Delta=\delta_{0} / \alpha, \psi=A \sqrt{\gamma L / \alpha}, t=$ $\alpha T / T_{R}$, and $\tau=T \sqrt{2 \alpha /\left(\left|\beta_{2} L\right|\right)}$, with $\alpha=\left(\theta+\alpha_{f} L\right) / 2$ being the total loss and $\delta_{0}=2 k \pi-\Phi_{0}$ is the detuning with respect to the nearest cavity resonance frequency. The coefficient $\eta= \pm 1$ is the sign of the second-order dispersion term. Here, we consider the anomalous dispersion case $\eta=-1$.

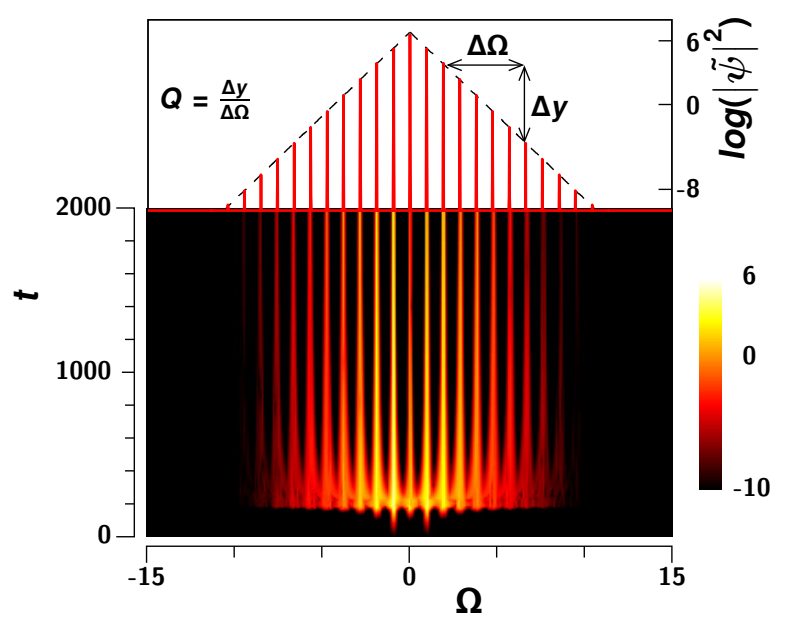

Fig. 2. (Bottom panel) Evolution of the intra-cavity field spectrum obtained by the numerical integration of Eq. (??) with a noisy initial condition. (Top panel) The asymptotic profile of the spectrum reached after $t=30000$. Parameters used in the simulations are $\Delta=1.3$, $\eta=-1$ and $I_{s}=1.046$.

Eq. (??) possesses solutions in the form of the homogeneous steady states (continuous waves) $\psi_{s}=\sqrt{I_{S}} \exp \left(i \phi_{s}\right)$ such that $S^{2}=$ $I_{S}\left[1+\left(\Delta-I_{S}\right)^{2}\right]$ and $\tan \left(\phi_{s}\right)=I_{s}-\Delta$. Therefore, it is straightforward task to check that the bifurcation diagram associated to this solution is monostable (or bistable) if the detuning parameter $\Delta<\sqrt{3}$ (or $\Delta>\sqrt{3}$ ). It is also easy to check that the homogeneous steady state is linearly unstable at $I_{s}=I_{\text {th }}=1$ leading to exponential growth of periodic perturbations with the most modulationally unstable sideband frequency given by $\Omega_{\mathrm{MI}}=\sqrt{\left(\Delta-2 I_{s}\right) / \eta}$.
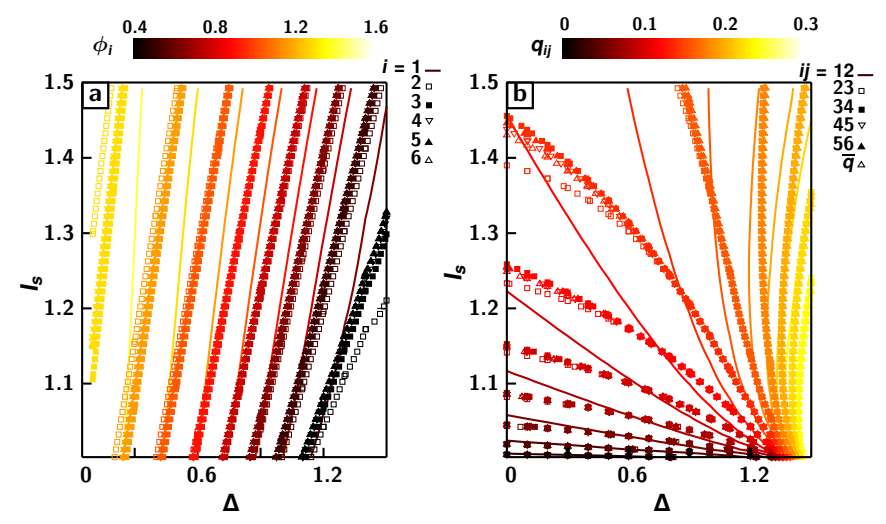

Fig. 3. (Color Online) Isolines corresponding to (a) the constant phase $\phi_{i}$ and (b) the constant ratio $q_{n m}=\sqrt{\left(a_{m}^{2}+b_{m}^{2}\right) /\left(a_{n}^{2}+b_{n}^{2}\right)}$ with $m=n+1$. These plots obtained by the numerical integration of the truncated system of fourteen equations limited to sixth harmonics. The isolines are equally spaced by $\Delta \phi=0.13 \mathrm{rad}$. They start from $\phi_{i}=0.4 \mathrm{rad}$ (black dots) and finish at $\phi_{i}=1.5 \mathrm{rad}$ (yellow dots). The curves in (b) are separated by $\Delta q_{m n}=0.02$. They start from $q_{m n}=0.005$ (black dots) and finish at $q_{m n}=0.25$ (yellow dots).

The typical evolution of the spectrum of the intra-cavity field is shown in Fig. ??. The lower panel shows the quick development of a wide spectrum of equidistant components typical for the advanced stage of modulation instability. The spread of the spectrum occurs through a four wave mixing process [?]. The asymptotic stationary spectrum shown in the upper panel has a characteristic triangular shape in the semi-logarithmic scale. Note that soliton combs may also appear in this systems. However, here we are dealing with frequency combs triangular spectrum that emerges from modulational instability in both monostable and bistable operating regimes. The spectral width of the harmonic sidebands separated by $\Delta \Omega=\Omega_{\mathrm{MI}}$ in this triangular spectrum is smaller than the free spectral range of the cavity. This differs the model considered here from other fiber optic cavities [? ]. Therefore, the temporal profile of the intra-cavity field can be written in terms of its Fourier components:

$$
\psi(t, \tau) \simeq \zeta(\tau)=\psi_{s}+A_{0}+\sum_{n=1}^{\infty}\left[A_{n}^{+} e^{i n \Delta \Omega \tau}+A_{n}^{-} e^{-i n \Delta \Omega \tau}\right],
$$

where each $A_{n}^{ \pm}$corresponds to the stationary amplitude associated to the $n$-th harmonic. Denoting by $Q$ the slope of the triangular spectrum as illustrated in Fig. ??, it is straightforward to check that

$$
\left|A_{n}^{ \pm}\right|=10^{\frac{Q \Delta \Omega}{2}}\left|A_{n-1}^{ \pm}\right|=q\left|A_{n-1}^{ \pm}\right|
$$

In order to verify this result, let us rewrite Eq. (??) in the following form:

$$
\psi(t, \tau) \simeq \zeta(t, \tau)=\psi_{s}+\sum_{n=0}^{\infty}\left[a_{n}(t)+i b_{n}(t)\right] \cos \left(n \Omega_{\mathrm{MI}} \tau\right),
$$

which is also known as the Galerkin expansion. Next, introducing this ansatz into Eq. (??), we find that the evolution of the amplitudes $a_{n}(\tau)$ and $b_{n}(\tau)$ are governed by two equations:

$$
\begin{aligned}
\frac{\partial a_{n}}{\partial t} & =\frac{1}{T_{\mathrm{MI}}} \int_{0}^{T_{\mathrm{MI}}} \Re\left\{\partial_{t} \psi(t, \tau)\right\} \cos \left(n \Omega_{\mathrm{MI}} \tau\right) d \tau, \\
\frac{\partial b_{n}}{\partial t} & =\frac{1}{T_{\mathrm{MI}}} \int_{0}^{T_{\mathrm{MI}}} \mathfrak{I}\left\{\partial_{t} \psi(t, \tau)\right\} \cos \left(n \Omega_{\mathrm{MI}} \tau\right) d \tau,
\end{aligned}
$$


where we have set $T_{\mathrm{MI}}=2 \pi / \Omega_{\mathrm{MI}}$. Hence, the stationary solutions of Eqs. (??) can be directly related to $A_{n}^{ \pm}$.

Truncating the Galerkin expansion to the order $n=6$, we solved the set of $2(n+1)$ equations in (??) numerically in order to find the asymptotic stationary solutions. For this purpose, we have used a fourth order fixed step Runge-Kutta scheme. Monte-Carlo method can also be used to find the stationary solutions of Eqs. (??). The results, in the parameter space $\left(\Delta, I_{S}\right)$, are shown in Fig. ??. Namely, Fig. ??a shows the lines of constant $\phi_{n}=\tan ^{-1}\left(b_{n} / a_{n}\right)$ while Fig. ??b shows the lines of constant $q_{n m}=\sqrt{\left(a_{m}^{2}+b_{m}^{2}\right) /\left(a_{n}^{2}+b_{n}^{2}\right)}$ with $m=n+1$. As can be seen from this figure, all components have the same phase except for the fundamental mode. In addition, the ratio of the modulus of two consecutive modes is constant in a large region of the parameters space. Thus, our numerical simulations demonstrate that the spectral components in Eq. (??) can be written in the form:

$$
\begin{aligned}
\zeta(\tau)= & \psi_{s}+A_{0} e^{i \phi_{0}}+A_{1} e^{i \phi_{1}} \cos \left(\Omega_{\mathrm{MI}} \tau\right) \\
& +\frac{A_{2}}{2} e^{i \phi_{2}} e^{2 i \Omega_{\mathrm{MI}} \tau} \sum_{k=0}^{\infty}\left(q e^{i \Omega_{\mathrm{MI}} \tau}\right)^{k} \\
& \frac{A_{2}}{2} e^{i \phi_{2}} e^{-2 i \Omega_{\mathrm{MI}} \tau} \sum_{k=0}^{\infty}\left(q e^{-i \Omega_{\mathrm{MI}} \tau}\right)^{k},
\end{aligned}
$$

where $A_{j}=\sqrt{a_{j}^{2}+b_{j}^{2}}$ with $j=\{0,1,2\}$. From Fig. ??b, it is also clear that the ratio $q$ between two consecutive harmonics satisfies the condition $q<1$. That is, the sums $\sum_{k=0}^{\infty}\left(q e^{ \pm i \Omega_{\mathrm{MI}} \tau}\right)^{k}$ are nothing but convergent geometric progressions and explicit expressions for them can be written. The resulting stationary periodic solutions of the Lugiato-Lefever equation are given by:

$$
\begin{aligned}
\psi(\tau)= & \psi_{s}+A_{0} e^{i \phi_{0}}+A_{1} e^{i \phi_{1}} \cos \left(\Omega_{\mathrm{MI}} \tau\right)+ \\
& A_{2} e^{i \phi_{2}} \frac{\cos \left(2 \Omega_{\mathrm{MI}} \tau\right)-q \cos \left(\Omega_{\mathrm{MI}} \tau\right)}{1+q^{2}-2 q \cos \left(\Omega_{\mathrm{MI}} \tau\right)} .
\end{aligned}
$$

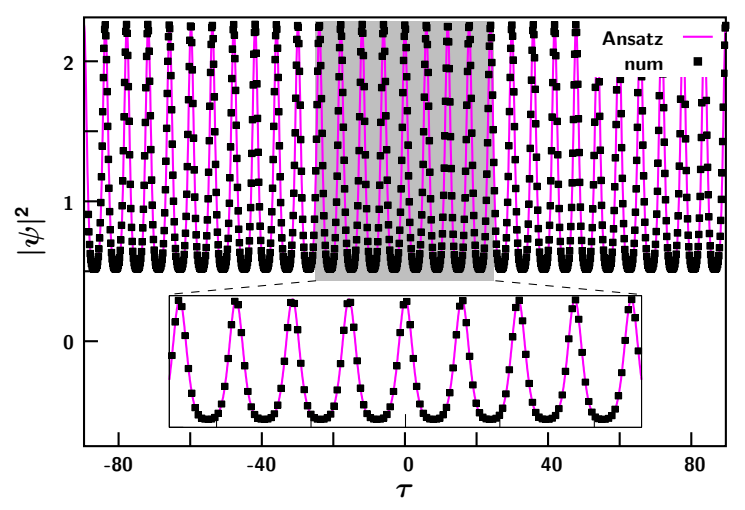

Fig. 4. (Color Online) Intracavity field profiles obtained from numerical integration of Eq. (??) (squares) and from the expression (??) (solid line). The insert shows a magnification of the shaded region located within the interval $|\tau| \leqslant 25$. The parameters are: $\Delta=1.300$, $I_{S}=1.203$.

Comparison of the solution given by (??) with the corresponding results of direct numerical simulations of LL equation is shown in Fig ??. As can be seen from this figure, the agreement between the two is excellent. An outstanding feature of the expression (??) is its ability to describe the periodic wave profiles which cannot be obtained by the standard weakly nonlinear multi-scale analysis. None of the existing nonlinear analytical methods can provide a similar result. Our system is not integrable, so the analytical exact solutions generally cannot be found. Indeed, we emphasize here that in the particular case when external pump and dissipation are small, LL equation reduces to damped and driven NLS equation. Although, even in this case no exact analytical solutions are known, they can be well approximated by means of perturbative methods (see for instance [? ]). Moreover, recently, exact solutions of the driven NLS equation, but without damping, have been obtained in [?] and very recently these solutions have been used to numerically reproduce a class of solutions appearing in Kerr-Comb systems where the damping is neglected [? ]. However, to the best of our knowledge, if the damping is present and not small, only numerical solutions are known. Let us mention, among others, the extensive work in [? ? ] where an interesting comparison of different numerical solutions obtained by varying the damping and the pump in a large set of parameters values is reported. In particular, it is shown that the solutions are very sensitive to the damping values, which makes hard any comparison of these solutions to the ones obtained without the damping term. On the other hand, it was shown, in [? ? ], that the equations involving order parameters that govern the evolution of the periodic states near the threshold are not valid above the value $\Delta=41 / 30 \simeq 1.3667$. In this case, the bifurcation describing the amplitude of the periodic state changes from super-critcal to sub-critical. In Fig ??, we have plotted the profile of the numerically computed solution and the results given by Eq. (??) for $\Delta=1.4$. This value of $\Delta$ is above the subcritical bifurcation threshold. This figure again shows an excellent agreement between the curve calculated from Eq. (??) and data from the numerical solutions. This result confirms that our present technique is free from the limitations considered in [? ? ]. We should also mention that Eq.(??) can, in principle, be applied to the experimental results shown in Fig.5(a) of the work [? ].

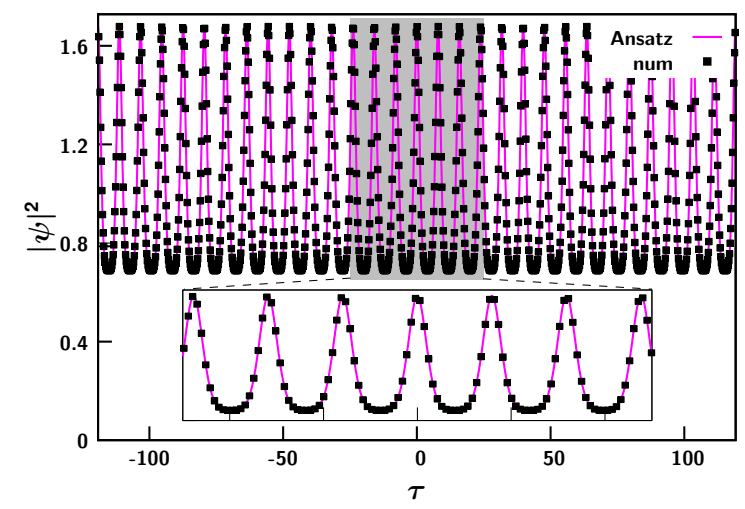

Fig. 5. (Color Online) Same as in Fig.?? except for the parameters used here: $\Delta=1.400, I_{s}=1.013$. This value of $\Delta$ is above the subcritical bifurcation threshold.

We would like to emphasise that even the results obtained from the significantly reduced system of ordinary differential equations, namely, when truncating the Garlerkin expansion in Eq. (??) to the 3rd order $(n=3)$, are also in reasonably good agreement with the numerical simulations. Let us denote as $q_{0}$ and $q_{1}$ the values obtained from truncating the system (??) to $n=6$ and to $n=3$, respectively. Then, the relative error $\delta q=\left(q_{0}-q_{1}\right) / q_{1}$ calculated in our routine is not greater than $6 \%$ within the whole parameter space we dealt with. This can be clearly seen from Fig. ??, where $\delta q$ is plotted in $\%$ as a function of the two experimentally accessible parameters $\Delta$ and $I_{s}$.

Remarkably, the expression (??) found in this work has wider range of applicability than in the above examples. In particular, it displays a bifurcation occurring when increasing the input parameter and holding the detuning fixed. The main feature of this bifurcation is that above the threshold the period of the solution deviates from the one obtained from 


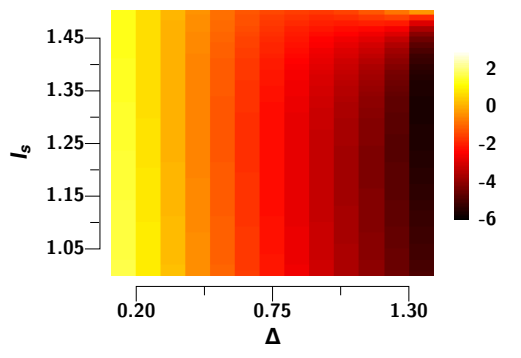

Fig. 6. (Color Online) Relative error $\delta q=100\left(q_{0}-q_{1}\right) / q_{1}$ in $\%$ (colour scale), with $q_{0}$ and $q_{1}$ being the values of $q$ obtained with truncation of Eqs.(??) at $n=6$ and $n=3$, respectively.

the linear stability analysis. This frequency deviation is accompanied with the change of the temporal profile and growth of additional local maxima as shown by the solid line in Fig. ??. Taking advantage of the triangular shape of the spectrum, we were able to fit this profile, by the following function:

$$
\psi=u_{0}+u_{1} \frac{1}{1+q^{2}-2 q \cos (\Delta \Omega \tau)} .
$$

This approximation is also reasonably accurate as validated by the stars in Fig. ?? calculated from Eq.(??). The work on precise characterisation of this bifurcation is in progress and the results will be published elsewhere. One more step could be an attempt to describe the birchromatic optical fields in the cavity [? ]. However, this is beyond our present tasks.

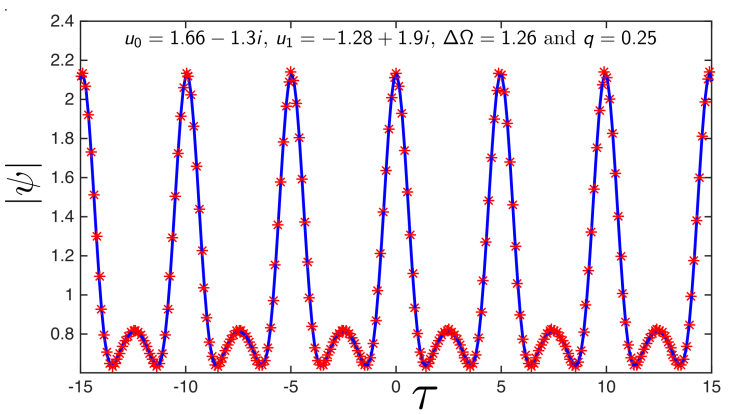

Fig. 7. Periodic solution observed in LL model (solid line) and the result of the fitting based on Eq. (??) (stars) for $I=2.0, \eta=-1.0$, $\Delta=1.3$.

In conclusion, we developed a method that allows us to write an analytic expression for intra-cavity periodic field profiles within the externally pumped Kerr-resonators. The method is based on empirical knowledge of the triangular shape of the frequency comb spectrum. The results obtained from this analytic expression are in good agreement with the outcome of the direct numerical simulations of the LugiatoLefever equation.

Z.L, S.C., M.T. acknowledge support from the ANR Blanc project Optiroc. N. A. acknowledges the support of the Australian Research Council (Discovery Project DP150102057)

\section{REFERENCES}

1. S. T. Cundiff and J. Ye, Rev. Mod. Phys., 75, 325 (2003).

2. N. R. Newbury, Nature Photonics, 5, 186 (2011).

3. J. L. Hall, Rev. Mod. Phys., 78, 1279 (2005);

4. T. W. Hänsch, Rev. Mod. Phys., 78, 1297 (2005).

5. Y. Okawachi, K. Saha, J. S. Levy, Y. H. Wen, M. Lipson and A. L. Gaeta, Opt. Lett., 36, 3398 (2011).
6. P. Parra-Rivas, D. Gomila, F. Leo, S. Coen, and L. Gelens, Opt. Lett., 39, 2971 (2014).

7. Y. K. Chembo, D. V. Strekalov, and N. Yu, Phys. Rev. Lett. 104, 103902 (2010).

8. G. Millot, S. Pitois, M. Yan, T. Hovhannisyan, A. Bendahmane, T. W. Hänsch and N. Picqué, Frequency-agile dual-comb spectroscopy, Nature Photonics, 10, 27 (2015).

9. C. Godey, I. V. Balakireva, A. Coillet, and Y. K. Chembo, Phys. Rev. A 89, 063814 (2014).

10. S. Coen, H. G. Randle, T. Sylvestre, and M. Erkintalo, Opt. Lett. 38, 37 (2013).

11. T. Herr, V. Brasch, J. D. Jost, C. Y. Wang, N. M. Kondratiev, M. L. Gorodetsky and T. J. Kippenberg, Nature Photonics, 8, 145 (2014).

12. V. Brasch, M. Geiselmann, T. Herr, G. Lihachev, M.H.P. Pfeiffer, M. L. Gorodetsky, T. J. Kippenberg, Science 351, 357 (2016).

13. N. Akhmediev and N. Devine, Science 351, 340 (2016).

14. S. Coen, M. Tlidi, P. Emplit, and M. Haelterman, Phys. Rev. Lett., 83, 2328 (1999).

15. S. Coulibaly, M. Taki, and M. Tlidi, Optics Express 22, 483 (2014).

16. F. Leo, S. Coen, P. Kockaert, S.-P. Gorza, P. Emplit, and M. Haelterman, Nature Photon., 4, 471 (2010)

17. M. Tlidi, L. Bahloul, L. Cherbi, A. Hariz, and S. Coulibaly, Phys. Rev. A 88, 035802 (2013).

18. A. Mussot, E. Louvergneaux, N. Akhmediev, F. Reynaud, L. Delage, and M. Taki, Optical Fiber Systems Are Convectively Unstable, Phys. Rev. Lett., 101, 113904 (2008).

19. S. Coen and M. Haelterman, Phys. Rev. Lett., 79, 4139 (1997).

20. G. Kozyreff, M. Tlidi, A. Mussot, E. Louvergneaux, M. Taki, and A. G. Vladimirov, Phys. Rev. Lett. 102, 043905 (2009).

21. F. Leo, A. Mussot, P. Kockaert, P. Emplit, M. Haelterman and M. Taki, Phys. Rev. Lett. 110, 104103 (2013).

22. N. Akhmediev and V. I. Korneev, Teor. Mat. Fiz. (USSR), 69, No 2, 189 (1986). [Translation: Theor. Math. Phys., 69, 1089 (1987)].

23. J. M. Dudley, G. Genty, F. Dias, B. Kibler, and N. Akhmediev, Optics Express 17, 21497 (2009).

24. N. Akhmediev, A. Ankiewicz, J. M. Soto-Crespo, and J. M. Dudley, Phys. Lett., A 375, 775 (2011).

25. M. Tlidi, A. Mussot, E. Louvergneaux, G. Kozyreff, A. G. Vladimirov, and M. Taki, Opt. Lett. 32, 662 (2007).

26. M. Haelterman, S. Trillo, and S. Wabnitz, Opt. Commun. 91, 401 (1992).

27. M. Taki et al., Physica D 40, 65-82 (1989)

28. Raju, T. S., et al.; J. of Phys. A: Mathematical and General 38, L271 (2005).

29. Renninger, W. H. and P. T. Rakich, Scientific Reports 6, 24742 (2016).

30. I Barashenkov and Y. Smirnov, Phys. Rev. E 54, 5707-5725 (1996).

31. P. Parra-Rivas, D. Gomila, M. A. Matías, S. Coen and L. Gelens, Dynamics of localized and patterned structures in the Lugiato-Lefever equation determine the stability and shape of optical frequency combs, Phys. Rev. A, 89, 043813 (2014).

32. L. A. Lugiato and R. Lefever, Phys. Rev. Lett. 58, 2209 (1987).

33. T. Herr, M. L. Gorodetsky, T. J. Kippenberg, Dissipative Kerr solitons in optical microresonators, In: Nonlinear optical cavity dynamics, Ed. Ph. Grelu, (Wiley-VCH, NY, 2016).

34. A. Bendahmane, J. Fatome, C. Finot, G. Millot and B. Kibler, Coherent and incoherent seeding of dissipative modulation instability in a nonlinear fiber ring cavity, Opt. Lett., 42, 251 (2017).

35. D. Ceoldo, A. Bendahmane, J. Fatome, G. Millot, T. Hansson, D. Modotto, S. Wabnitz and B. Kibler, Multiple four-wave mixing and Kerr combs in a bichromatically pumped nonlinear fiber ring cavity, Opt. Lett., 41, 5462 (2016). 